\title{
Aperture corrections for disk galaxy properties derived from the CALIFA survey
}

\section{Balmer emission lines in spiral galaxies}

\author{
J. Iglesias-Páramo ${ }^{1,2}$, J. M. Vílchez ${ }^{1}$, L. Galbany ${ }^{3}$, S. F. Sánchez ${ }^{1,2}$, F. F. Rosales-Ortega ${ }^{4}$, D. Mast ${ }^{2}$, R. García-Benito ${ }^{1}$, \\ B. Husemann ${ }^{5}$, J. A. L. Aguerri ${ }^{6,7}$, J. Alves ${ }^{8}$, S. Bekeraité ${ }^{5}$, J. Bland-Hawthorn ${ }^{9}$, C. Catalán-Torrecilla ${ }^{10}$, \\ A. L. de Amorim ${ }^{1,11}$, A. de Lorenzo-Cáceres ${ }^{6,7}$, S. Ellis ${ }^{12}$, J. Falcón-Barroso ${ }^{6,7}$, H. Flores $^{13}$, E. Florido ${ }^{14,15}$, \\ A. Gallazzi ${ }^{16,17}$, J. M. Gomes ${ }^{18}$, R. M. González Delgado ${ }^{1}$, T. Haines ${ }^{19}$, J. D. Hernández-Fernández ${ }^{20}$, C. Kehrig ${ }^{1}$, \\ A. R. López-Sánchez ${ }^{12,21}$, M. Lyubenova ${ }^{22}$, R. A. Marino ${ }^{23}$, M. Mollá ${ }^{24}$, A. Monreal-Ibero ${ }^{1}$, A. Mourão ${ }^{3}$, \\ P. Papaderos ${ }^{18}$, M. Rodrigues ${ }^{27}$, P. Sánchez-Blázquez ${ }^{25}$, K. Spekkens ${ }^{26}$, V. Stanishev ${ }^{3}$, G. van de Ven ${ }^{22}$, C. J. Walcher ${ }^{5}$, \\ L. Wisotzki ${ }^{5}$, S. Zibetti ${ }^{17}$, and B. Ziegler ${ }^{8}$
}

(Affiliations can be found after the references)

Received 13 March 2013 / Accepted 4 April 2013

\begin{abstract}
This work investigates the effect of the aperture size on derived galaxy properties for which we have spatially-resolved optical spectra. We focus on some indicators of star formation activity and dust attenuation for spiral galaxies that have been widely used in previous work on galaxy evolution. We investigated 104 spiral galaxies from the CALIFA survey for which 2D spectroscopy with complete spatial coverage is available. From the $3 \mathrm{D}$ cubes we derived growth curves of the most conspicuous Balmer emission lines $(\mathrm{H} \alpha, \mathrm{H} \beta)$ for circular apertures of different radii centered at the galaxy's nucleus after removing the underlying stellar continuum. We find that the $\mathrm{H} \alpha$ flux $(f(\mathrm{H} \alpha))$ growth curve follows a well-defined sequence with aperture radius that shows a low dispersion around the median value. From this analysis, we derived aperture corrections for galaxies in different magnitude and redshift intervals. Once stellar absorption is properly accounted for, the $f(\mathrm{H} \alpha) / f(\mathrm{H} \beta)$ ratio growth curve shows a smooth decline, pointing toward the absence of differential dust attenuation as a function of radius. Aperture corrections as a function of the radius are provided in the interval $[0.3,2.5] R_{50}$. Finally, the $\mathrm{H} \alpha$ equivalent-width $(\mathrm{EW}(\mathrm{H} \alpha))$ growth curve increases with the size of the aperture and shows a very high dispersion for small apertures. This prevents us from using reliable aperture corrections for this quantity. In addition, this result suggests that separating star-forming and quiescent galaxies based on observed $\mathrm{EW}(\mathrm{H} \alpha)$ through small apertures will probably result in low $\mathrm{EW}(\mathrm{H} \alpha)$ star-forming galaxies begin classified as quiescent.
\end{abstract}

Key words. techniques: spectroscopic - galaxies: evolution - surveys - galaxies: ISM

\section{Introduction}

The advent of large area surveys has enormously advanced our knowledge of galaxy formation and evolution in recent decades. In particular, the surveys consisting of imaging and single fiber spectroscopy (e.g. 2dFGRS, Folkes et al. 1999; SDSS, York et al. 2000; GAMA, Driver et al. 2011) have provided invaluable information on redshift as well as other galactic properties such as star formation rates (SFR) or metallicities, via the intensities of the most conspicuous emission lines. However, aperture effects are always present in such studies because of the limited (and variable with redshift) coverage of the individual galaxies. Consequently, a fraction of the total flux at all wavelengths is lost and, to date, no recipe to correct for this effect has proved satisfactory, although several studies of aperture effects have been carried out (e.g. Brinchmann et al. 2004; Ellis et al. 2005; Kewley et al. 2005; Salim et al. 2007; Kronberger et al. 2008; Gerssen et al. 2012; Zahid et al. 2013; Hopkins et al. 2013).

In practice, there are two main problems: one is the size of the projection on the sky of the aperture relative to the physical dimensions of the galaxy. The second one, which mainly affects single-fiber (or very small aperture) spectrographs (e.g. $2^{\prime \prime}$ for 2dFGRS and GAMA; 3" for SDSS), is the precise position of the aperture relative to the galaxy center. This aperture effect has implications for spectral stacking studies, since the fraction of a galaxy covered by a fixed aperture varies with redshift. Therefore using a realistic aperture correction is a sine qua non condition to carry out the analysis of aperture spectra of galaxies at different epochs, to derive their fundamental properties, and to discuss their evolution.

Gerssen et al. (2012) have recently carried out a study of a reduced sample of SDSS star-forming galaxies using the VIMOS integral field spectrograph (Le Fèvre et al. 2003) to map (with full spatial coverage) some properties related to star formation diagnostics. These authors found a high dispersion when comparing their results with color-based SDSS extrapolations. This suggests that full spatial coverage is needed to produce proper corrections for emission line intensities, and large samples of galaxies covering all morphological types are required.

The Calar Alto Legacy Integral Field Area Survey (CALIFA; Sánchez et al. 2012a) is observing a statistically well-defined sample of 600 galaxies in the local Universe with the Potsdam Multi Aperture Spectrograph in the PPAK mode (Roth et al. 2005). Galaxies have been selected from the SDSS-DR7 catalog 
(Abazajian et al. 2009) to fulfill the following conditions: (1) they fall in the redshift range $0.005<z<0.03$; and (2) their isophotal diameters in the $r^{\prime}$ band (isoA_r in SDSS-DR7) are in the range $45^{\prime \prime}<i$ soA_r $r<80^{\prime \prime}$. Combining these two criteria results in a sample of galaxies that fall completely within the PPAK field-of-view and covers all relevant emission lines with a single spectral setup. The CALIFA galaxies completely sample the color-magnitude diagram down to $M_{r}=-19 \mathrm{mag}$ (see Sánchez et al. 2012a). In summary, the data provided by CALIFA are well-suited to testing the aperture effects discussed above, thanks to complete spectral and spatial coverage of the sample galaxies, and providing aperture correction factors as a function of aperture size for quantities that vary smoothly with the latter. In our analysis, we adopt the following cosmological parameters: $H_{0}=72 \mathrm{~km} \mathrm{~s}^{-1} \mathrm{Mpc}^{-1}, \Omega_{\mathrm{M}}=0.27$ and $\Omega_{\lambda}=0.73$.

\section{Aperture corrections}

The basic tools for this process are the CALIFA reduced cubes from the DR1 sample (Husemann et al. 2013). Our starting point is a larger sample containing 258 galaxies including those of DR1 and some others not yet released to the community. The survey sample comprises $24.4 \%$ E-S0 and $75.6 \%$ Sa-Sm galaxies. We removed the galaxies suspected of being Active Galactic Nuclei (AGN) according to several criteria based on optical spectra and radio data following the criteria of Best et al. (2012), Kewley et al. (2001, 2006), and Cid Fernandes et al. (2011). We also removed galaxies with morphological types earlier than S0a since we want to focus only on spiral galaxies. To keep only galaxies completely observed within the PPAK field-of-view, we rejected all galaxies for which $2.5 R_{50} \geq 36^{\prime \prime}$, where $R_{50}$ is the radius containing $50 \%$ of the Petrosian flux in the $r^{\prime}$ band (petroR50_ $r$ in SDSS-DR7). After testing several options such us half-light radii from circular and elliptical growth curve analysis, we decided to use petroR50_r as our scale because it results in the largest sample of galaxies fulfilling our requirements, and has the least-dispersed profiles for the growth curves to be discussed later. This left us with a sample of 104 galaxies with morphological types from Sa to Sm completely covered by the PPAK field-of-view. The median values ( $1 \sigma$ confidence interval) of the stellar mass, SFR (from dustcorrected $\mathrm{H} \alpha$ luminosities), $\mathrm{EW}(\mathrm{H} \alpha)$, and $f(\mathrm{H} \alpha) / f(\mathrm{H} \beta)$ distributions of the sample are $\log M^{*} / M_{\odot}=10.25([9.65,10.85])$, $\log S F R /\left(M_{\odot} \mathrm{yr}^{-1}\right)=0.45([-0.06,0.79]), E W(\mathrm{H} \alpha) / \AA=16.91$ $([6.39,28.56])$, and $f(\mathrm{H} \alpha) / f(\mathrm{H} \beta)=4.75([4.00,5.89])$. We produced stacked spectra for each galaxy by adding the emission from the spaxels within circular apertures centered at the galaxy's nucleus and with radii increasing from $1.5^{\prime \prime}$ by steps of $1.5^{\prime \prime}$ up to a maximum radius completely covering the hexagonal PPAK aperture. Then we subtracted the stellar continuum to each of our stacked spectra by using the FIT3D code (Sánchez et al. 2011), which yielded a set of spectra of the ionized gas emission. Finally, the fluxes of the emission lines within each aperture were extracted from two independent Gaussian fits with the IDL-based routine MPFITEXPR (Markwardt 2009), one for $\mathrm{H} \beta$ and another one for the triplet $\mathrm{H} \alpha+[\mathrm{NII}] \lambda 6548,6583 \AA$. A more detailed description of this point will be included in a forthcoming paper.

\section{Results}

We illustrate our results with the aperture corrections for three observables: $f(\mathrm{H} \alpha)$, the $f(\mathrm{H} \alpha) / f(\mathrm{H} \beta)$ ratio, and the $\mathrm{EW}(\mathrm{H} \alpha)$,

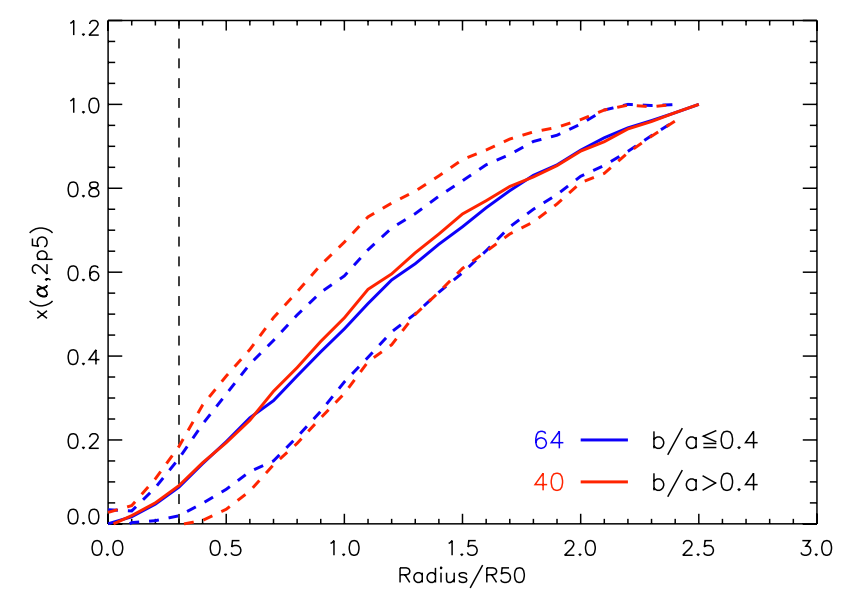

Fig. 1. Growth curve of $f(\mathrm{H} \alpha)$ normalized to $f(\mathrm{H} \alpha)$ at $2.5 R_{50}$, $x(\alpha, 2 p 5)$, as a function of the radius of the aperture. Bold red (blue) lines correspond to the median values for galaxies with $b / a>0.4$ $(b / a<0.4)$. Dashed red and blue lines correspond to $1 \sigma$ deviations from the median curve. Numbers indicate the size of each subsample. The vertical dashed line corresponds to $0.3 R_{50}$, which on average corresponds to the FWHM of the CALIFA psf for our sample.

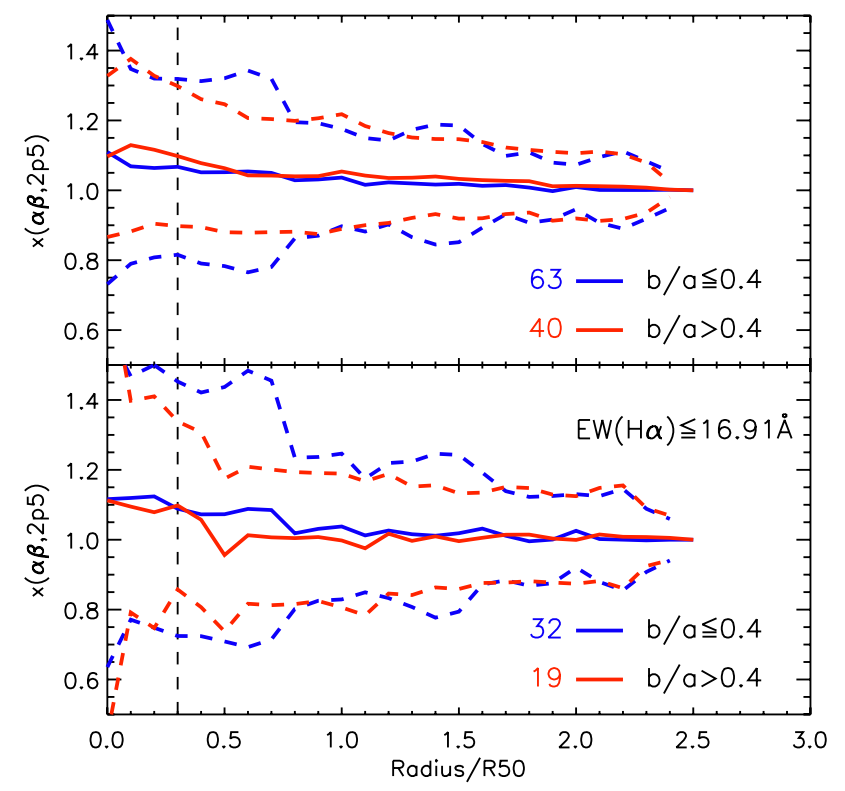

Fig. 2. Top: growth curve of $f(\mathrm{H} \alpha) / f(\mathrm{H} \beta)$ normalized to $f(\mathrm{H} \alpha) / f(\mathrm{H} \beta)$ at $2.5 R_{50}, x(\alpha \beta, 2 p 5)$, as a function of the radius of the aperture. Lines and colors as in Fig. 1. For one of the galaxies the $\mathrm{H} \beta$ emission line was not detected in all apertures and was not included. Bottom: same as top panel restricted to galaxies with $E W(\mathrm{H} \alpha) \leq 16.91 \AA$.

shown in Figs. 1 to 3 . The figures show the median profiles of the growth curves corresponding to these three quantities normalized to the value measured within an external isophote containing most of the optical light of the galaxy. We normalized the profiles to the value measured at $2.5 R_{50}$ since this is the typical value covered by the PPAK field-of-view for the CALIFA sample. This aperture, $2.5 R_{50}$, encloses on average $\simeq 90 \%$ of the total $\mathrm{H} \alpha$ flux of spiral galaxies ${ }^{1}$.

\footnotetext{
1 Estimated by adding the contribution of all the spaxels of the PPAK aperture for each galaxy of the sample to the $\mathrm{H} \alpha$ emission flux. This must be taken as a rough estimate since the largest galaxies in the sample show $\mathrm{H} \alpha$ emission beyond the PPAK aperture.
} 
J. Iglesias-Páramo et al.: Aperture corrections for disk galaxy properties derived from the CALIFA survey

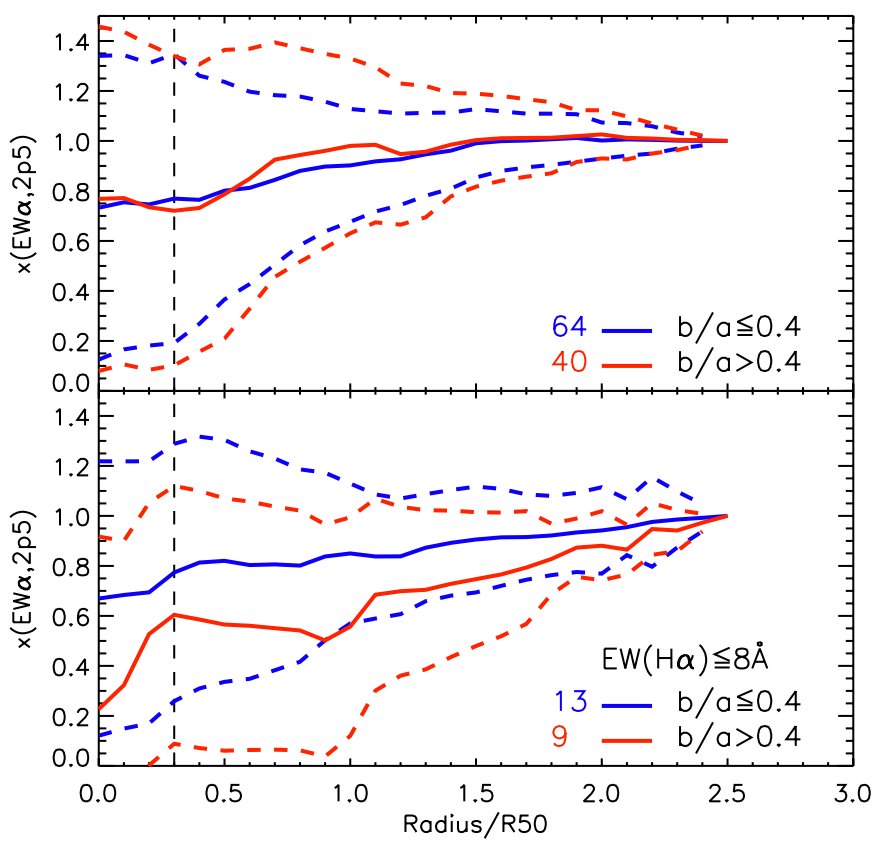

Fig. 3. Top: growth curve of $\mathrm{EW}(\mathrm{H} \alpha)$ normalized to $\mathrm{EW}(\mathrm{H} \alpha)$ at $2.5 R_{50}$, $x(E W \alpha, 2 p 5)$, as a function of the radius. Bottom: same as top panel restricted to galaxies with $E W(\mathrm{H} \alpha) \leq 8 \AA$ at $2.5 R_{50}$.

Figure 1 shows the median $f(\mathrm{H} \alpha)$ growth curve normalized to $f(\mathrm{H} \alpha)$ at $2.5 R_{50}, x(\alpha, 2 p 5)$. We split our sample according to the axis ratio to take into account the effect that the inclination of the galaxy may have on the values measured through circular apertures. For this, we use the isophotal axis in the $r^{\prime}$ band $i s o A \_r$ and $i s o B \_r$ from SDSS-DR7. This choice gives average values of $\left\langle i s o B \_r / i s o A \_r\right\rangle=0.27$ and 0.53 , respectively, for each subsample. We verified that other choices like the semi-minor to semi-major axis from elliptical growth curve and light momentum analysis yield basically the same average inclination distributions as our selection. As can be seen, the average curves for both ranges of inclination are quite similar within the $1 \sigma$ uncertainties. The low thickness of the ionized gas disks, as estimated from the low-velocity dispersion of the HII regions ( $\approx 10 \mathrm{~km} \mathrm{~s}^{-1}$, Relaño et al. 2005), suggests that no significant differences in the $\mathrm{H} \alpha$ growth curves of face-on and edge-on galaxies should be expected. However, a circular aperture covers different parts of the disk for face-on and edge-on galaxies. Thus, the similarity found for both curves might partly arise because the average inclination values of our subsamples are far from the extreme face-on and edge-on cases and close enough to each other, although we verified that even the curves restricted to galaxies with the most extreme values in our sample are not significantly different from each other. A physically motivated study of the $\mathrm{H} \alpha$ spatial distribution for face-on and edge-on galaxies is beyond the scope of this work and will be part of ongoing work devoted to the SFR in CALIFA galaxies. Similarly, aperture corrections as a function of Hubble type require a larger sample and will be part of future papers on this topic. We list in Table 1 the median growth curve and the $1 \sigma$ uncertainties of $f(\mathrm{H} \alpha)$ as a function of the aperture radius. Because galaxies with low and high inclination show very similar results, these values correspond to our complete working sample.

An interesting problem is the effect of the Full Width at Half Maximum (FWHM) of the CALIFA Point Spread Function (PSF, $3.6^{\prime \prime}$ ) on the growth curves of the emission lines.
Table 1. $x(\alpha, 2 p 5)$ and $x(\alpha \beta, 2 p 5)$ and their $1 \sigma$ deviations as a function of the size of the circular aperture normalized to $R_{50}$.

\begin{tabular}{lcccc}
\hline \hline$r / R_{50}$ & $x(\alpha, 2 p 5)$ & $(\sigma)$ & $x(\alpha \beta, 2 p 5)$ & $(\sigma)$ \\
\hline 0.3 & 0.091 & $(0.074)$ & 1.078 & $(0.221)$ \\
0.5 & 0.194 & $(0.119)$ & 1.052 & $(0.240)$ \\
0.7 & 0.301 & $(0.156)$ & 1.042 & $(0.234)$ \\
0.9 & 0.419 & $(0.158)$ & 1.034 & $(0.163)$ \\
1.1 & 0.530 & $(0.146)$ & 1.028 & $(0.136)$ \\
1.3 & 0.629 & $(0.130)$ & 1.021 & $(0.145)$ \\
1.5 & 0.718 & $(0.117)$ & 1.026 & $(0.154)$ \\
1.7 & 0.798 & $(0.101)$ & 1.020 & $(0.093)$ \\
1.9 & 0.854 & $(0.079)$ & 1.009 & $(0.088)$ \\
2.1 & 0.917 & $(0.070)$ & 1.009 & $(0.129)$ \\
2.3 & 0.961 & $(0.039)$ & 1.003 & $(0.086)$ \\
2.5 & 1.000 & - & 1.000 & - \\
\hline
\end{tabular}

Table 2. Redshift and absolute magnitude ranges at which the aperture corrections listed in Table 1 are valid for SDSS and SAMI data.

\begin{tabular}{lcc}
\hline \hline Redshift & \multicolumn{2}{c}{ Absolute magnitude range } \\
& SDSS & SAMI \\
\hline 0.02 & $-18 \lesssim M_{r}$ & $-22.5 \lesssim M_{r} \lesssim-16.5$ \\
0.04 & $-20.5 \lesssim M_{r}$ & $-22 \lesssim M_{r} \lesssim-18.5$ \\
0.06 & $-21.5 \lesssim M_{r} \lesssim-16$ & $M_{r} \lesssim-20$ \\
0.08 & $-22 \lesssim M_{r} \lesssim-16.5$ & $M_{r} \lesssim-21$ \\
0.10 & $-22.5 \lesssim M_{r} \lesssim-17$ & $M_{r} \lesssim-21.5$ \\
0.14 & $-22.5 \lesssim M_{r} \lesssim-18$ & \\
0.18 & $-22.5 \lesssim M_{r} \lesssim-19$ & \\
\hline
\end{tabular}

Trujillo et al. (2001) demonstrated that the parameters of a Sérsic profile are affected by the seeing. For exponential disks, which correspond to a $n=1$ profile, the central intensity and effective radius are recovered within $20 \%$ of their true value, disregarding the ellipticity as far as $F W H M / r_{\text {eff }} \leq 0.3$. This result prevents the use of aperture corrections below this limit since they could be severely affected by seeing. Given that the average value of $R_{50}$ for our sample is $\approx 10^{\prime \prime}$, the range of validity of our aperture correction is valid for radii $r / R_{50} \gtrsim 0.3$. Another limitation is the fact that the observed size of the galaxies decreases as we move to higher redshifts, and this imposes both a lower and an upper limit to the range of magnitudes for which our correction can be applied. Based on average measurements of $R_{50}$ for spiral galaxies from SDSS, we derived the validity ranges of the aperture corrections at different redshifts. In Table 2, we show these ranges for two representative projects providing spectra with different aperture sizes: SDSS (single-fiber, $\varnothing=3^{\prime \prime}$ ) and SAMI (multi-fiber bundle, $\varnothing=15^{\prime \prime}$, Bryant et al. 2011). In both cases the lower and upper magnitude limits correspond to the magnitudes at which $r_{\text {aper }} / R_{50} \geq 0.3$ and $r_{\text {aper }} / R_{50} \leq 2.5$, respectively ${ }^{2}$, based on their average sizes at the different redshift proved with a sample of SDSS-DR7 galaxies. The applicability of the aperture corrections is meaningful for individual galaxies within the limits listed in Table 2, thus we do not recommend to apply average corrections to large samples of galaxies

2 There is another restriction on the upper magnitude limit due to the effect of the PSF on the determination of $R_{50}$. This upper limit corresponds to the magnitude at which $F W H M \approx R_{50}$ and it must be applied when it is more restrictive than the one given in table 2 for each redshift. In the particular case of SDSS images, $R_{50}$ are not corrected for the psf such that this limit can become relevant at high redshifts. A discussion on the limits of the reliability of $R_{50}$ at different redshifts can be found in González-Pérez et al. (2011). 
to correct quantities such as the star formation density from observed $\mathrm{H} \alpha$ luminosities.

Figure 2 (top panel) shows the median $f(\mathrm{H} \alpha) / f(\mathrm{H} \beta)$ ratio growth curve normalized to $2.5 R_{50}, x(\alpha \beta, 2 p 5)$, again split for low- and high-inclination galaxies. Both curves show a smooth decline from a central value of $\simeq 1.1$ to 1.0 at the normalization radius and no significant difference with the inclination of the galaxies. The median growth curve and the $1 \sigma$ uncertainty for the whole sample are listed in Table 1 . The very smooth decline of $x(\alpha \beta, 2 p 5)$ supports the earlier result that the dust attenuation shows little dependence on the radial distance (Sánchez et al. 2012b). We examined the effect of non-negligible residual $\mathrm{H} \beta$ absorption after subtracting the underlying stellar continuum by restricting the analysis to galaxies with $E W(\mathrm{H} \alpha) \leq 16.9 \AA$ (see lower panel of Fig. 2). Clearly, this growth curve is consistent within the uncertainties with the one corresponding to the whole sample, which suggests that our $\mathrm{H} \beta$ fluxes, and thus the $f(\mathrm{H} \alpha) / f(\mathrm{H} \beta)$ ratio, are on average free from this effect.

Figure 3 (top panel) shows the median $\operatorname{EW}(\mathrm{H} \alpha)$ growth curve, $x(E W \alpha, 2 p 5)$, which as in previous cases is similar for galaxies with low- and high-inclination. This curve grows with radius, ranging from a central value of $\simeq 0.7$ to 1 at the normalization radius. The high dispersion of this quantity measured at small apertures $\left(\sigma>0.3\right.$ at $\left.r / R_{50} \leq 1.3\right)$ is remarkable. The observed dispersion at small radii arises because $\mathrm{EW}(\mathrm{H} \alpha)$ is an intensive quantity (in contrast to $\mathrm{H} \alpha$ or $\mathrm{H} \beta$ fluxes which are extensive quantities), and thus very low or high values at small radii can be measured because of features such as circumnuclear rings of star formation or nuclear starbursts. This high dispersion at small radii rules out a reliable aperture correction for the $\operatorname{EW}(\mathrm{H} \alpha)$. The main implication of the high dispersion at small radii is the possibility that star-forming galaxies observed through a small aperture are misclassified as quiescent if the classification is based only on an extrapolation of the central value of the $\operatorname{EW}(\mathrm{H} \alpha)$. To illustrate this, we show $x(\mathrm{EW} \alpha, 2 p 5)$ in the bottom panel of Fig. 3 as a function of radius restricted to galaxies with $E W(\mathrm{H} \alpha) \leq 8 \AA$. The curve of the low-inclination galaxies is clearly below that of the high-inclination ones, which follows a trend similar to that of the whole sample. As an example, according to this plot a face-on spiral galaxy with (total) $E W(\mathrm{H} \alpha)=6 \AA$ and observed through an aperture covering less than $R_{50}$ will have a $\simeq 50 \%$ chance of showing (observed) $E W(\mathrm{H} \alpha) \leq 3 \AA$, and thus of being classified as quiescent depending on the adopted limit (previous works separate active and quiescent galaxies at $E W(\mathrm{H} \alpha) \simeq 2-4 \AA$, see Cid Fernandes et al. 2010). This effect is less dramatic for edge-on galaxies.

We conclude that the median $f(\mathrm{H} \alpha)$ growth curve of spiral galaxies is well defined and shows a low dispersion for a range of aperture sizes between $0.3 R_{50}$ and $2.5 R_{50}$. The $f(\mathrm{H} \alpha) / f(\mathrm{H} \beta)$ ratio growth curve presents a smooth behavior that suggests a low dependence of the dust attenuation with radius. Median aperture corrections for this quantity are also meaningful. The observed high dispersion around the median $\mathrm{EW}(\mathrm{H} \alpha)$ growth curve excludes deriving a reliable aperture correction especially at small radii. This high dispersion is a caveat in using the $\mathrm{EW}(\mathrm{H} \alpha)$ as the criterion to classify star-forming and quiescent galaxies for which the size of the aperture is $\lesssim R_{50}$, because it might result in misclassification of low $\mathrm{EW}(\mathrm{H} \alpha)$ galaxies. We finally add that this paper presents only the tip of the iceberg. Aperture effects are widely mentioned but rarely understood. Their influence can seriously affect scientific results through systematic errors (Bland-Hawthorn et al. 2011). Forthcoming papers will be devoted to providing a complete set of diagnostics involving other indicators of SFR and/or abundances, and to applying these results to existing datasets.

Acknowledgements. This study made use of the data provided by the Calar Alto Legacy Integral Field Area (CALIFA) survey (http://califa.caha. es/). The CALIFA collaboration would like to thank the IAA-CSIC and MPIA-MPG as major partners of the observatory, and CAHA itself, for the unique access to telescope time and support in manpower and infrastructures. The CALIFA collaboration also thanks the CAHA staff for the dedication to this project. Based on observations collected at the Centro Astronómico Hispano Alemán (CAHA) at Calar Alto, operated jointly by the Max-Planck-Institut für Astronomie and the Instituto de Astrofísica de Andalucía (CSIC). We thank the Viabilidad, Diseño, Acceso y Mejora funding program ICTS-2009-10, for supporting the initial developement of this project. J.I.-P., J.V.M., C.K. and A.M.I. thank the Spanish PNAYA projects Estallidos AYA2010-21887-C04-01. R.A.M. was funded by the Spanish programme of International Campus of Excellence Moncloa (CEI). J.F.-B. acknowledges support from the Ramón y Cajal programme by the Spanish Ministry of Economy and Competitiveness (MINECO). This work has been supported by the Programa Nacional de Astronomía y Astrofísica of MINECO, under grants AYA2010- 21322-C03-01 and AYA201021322-C03-02. L.G. and V.S. acknowledge financial support from Fundação para a Ciência e a Tecnologia (FCT) under program Ciência 2008 and the research grant PTDC/CTE-AST/112582/2009. The Dark Cosmology Centre is funded by the Danish National Research Foundation. A.G. acknowledges funding from the European Union Seventh Framework Programme (FP7/20072013) under grant agreement No. 267251. This research has made use of the NASA/IPAC Extragalactic Database (NED) which is operated by the Jet Propulsion Laboratory, California Institute of Technology, under contract with the National Aeronautics and Space Administration.

\section{References}

Abazajian, K. N., Adelman-McCarthy, J. K., Agüeros, M. A., et al. 2009, ApJS, 182,543

Best, P. N., \& Heckman, T. M. 2012, MNRAS, 421, 1569

Bland-Hawthorn, J., Bryant, J., Robertson, G., et al. 2011, Optics Express, 19, 2649

Brinchmann, J., Charlot, S., White, S. D. M., et al. 2004, MNRAS, 351, 1151

Bryant, J. J., O'Byrne, J. W., Bland-Hawthorn, J., \& Leon-Saval, S. G. 2011, MNRAS, 415, 2173

Cid Fernandes, R., Stasińska, G., Schlickmann, M. S., et al. 2010, MNRAS, 403, 1036

Cid Fernandes, R., Stasińska, G., Mateus, A., \& Vale Asari, N. 2011, MNRAS, 413, 1687

Driver, S. P., Hill, D. T., Kelvin, L. S., et al. 2011, MNRAS, 413, 971

Ellis, S. C., Driver, S. P., Allen, P. D., et al. 2005, MNRAS, 363, 1257

Folkes, S., Ronen, S., Price, I., et al. 1999, MNRAS, 308, 459

Gerssen, J., Wilman, D. J., \& Christensen, L. 2012, MNRAS, 420, 197

González-Pérez, V., Castander, F. J., \& Kauffmann, G. 2011, MNRAS, 411, 1151

Hopkins, A. M., Driver, S. P., Brough, S., et al. 2013, MNRAS, 430, 2047

Husemann, B., Jahnke, K., Sánchez, S. F., et al. 2013, A\&A, 549, A87

Kewley, L. J., Dopita, M. A., Sutherland, R. S., Heisler, C. A., \& Trevena, J. 2001, ApJ, 556, 121

Kewley, L. J., Jansen, R. A., \& Geller, M. J. 2005, PASP, 117, 227

Kewley, L. J., Groves, B., Kauffmann, G., \& Heckman, T. 2006, MNRAS, 372, 961

Kronberger, T., Kapferer, W., Unterguggenberger, S., Schindler, S., \& Ziegler, B. L. 2008, A\&A, 483, 783

Le Fèvre, O., Saisse, M., Mancini, D., et al. 2003, Proc. SPIE, 4841, 1670

Markwardt, C. B. 2009, Astronomical Data Analysis Software and Systems XVIII, 411, 251

Relaño, M., Beckman, J. E., Zurita, A., Rozas, M., \& Giammanco, C. 2005, A\&A, 431, 235

Roth, M. M., Kelz, A., Fechner, T., et al. 2005, PASP, 117, 620

Salim, S., Rich, R. M., Charlot, S., et al. 2007, ApJS, 173, 267

Sánchez, S. F., Rosales-Ortega, F. F., Kennicutt, R. C., et al. 2011, MNRAS, 410, 313

Sánchez, S. F., Kennicutt, R. C., Gil de Paz, A., et al. 2012a, A\&A, 538, A8

Sánchez, S. F., Rosales-Ortega, F. F., Marino, R. A., et al. 2012b, A\&A, 546, A2

Trujillo, I., Aguerri, J. A. L., Cepa, J., \& Gutiérrez, C. M. 2001, MNRAS, 321, 269

York, D. G., Adelman, J., Anderson, J. E., Jr., et al. 2000, AJ, 120, 1579

Zahid, H. J., Yates, R. M., Kewley, L. J., \& Kudritzki, R. P. 2013, ApJ, 763, 92 
1 Instituto de Astrofísica de Andalucía - CSIC, 18008 Granada, Spain e-mail: jiglesias@iaa.es

2 Centro Astronómico Hispano Alemán, 04004 Almería, Spain

3 CENTRA Centro Multidisciplinar de Astrofísica, Instituto Superior Técnico, Av. Rovisco Pais 1, 1049-001 Lisbon, Portugal

${ }^{4}$ Instituto Nacional de Astrofísica, Óptica y Electrónica, Luis E. Erro 1, 72840 Tonantzintla, Puebla, Mexico

5 Leibniz-Institut für Astrophysik Potsdam (AIP), An der Sternwarte 16, 14482 Potsdam, Germany

6 Instituto de Astrofísica de Canarias, vía Láctea s/n, La Laguna, Tenerife, Spain

7 Departamento de Astrofísica, Universidad de La Laguna, 38205 La Laguna, Tenerife, Spain

${ }^{8}$ University of Vienna, Department of Astrophysics, Türkenschanzstr. 17, 1180 Vienna, Austria

9 Sydney Institute for Astronomy, School of Physics A28, University of Sydney, NSW 2006, Australia

10 Departamento de Astrofísica y CC. de la Atmósfera, Universidad Complutense de Madrid, 28040 Madrid, Spain

11 Departamento de Física, Universidade Federal de Santa Catarina, PO Box 476, 88040-900 Florianpolis, SC, Brazil

12 Australian Astronomical Observatory, 105 Delhi Rd., North Ryde, NSW 1670, Australia

13 GEPI, Observatoire de Paris, CNRS-UMR8111, Univ. Paris-Diderot, 5 place Janssen, 92195 Meudon, France

14 Departamento de Física Teórica y del Cosmos, University of Granada, Facultad de Ciencias (Edificio Mecenas), 18071 Granada, Spain
15 Instituto Carlos I de Física Teórica y Computación, 18071 Granada, Spain

16 Dark Cosmology Centre, Niels Bohr Institute, University of Copenhagen, Juliane Mariesvej 30, 2100 Copenhagen, Denmark

17 INAF-Osservatorio Astrofisico di Arcetri, Largo Enrico Fermi 5, 50125 Firenze, Italy

${ }^{18}$ Centro de Astrofísica and Faculdade de Ciências, Universidade do Porto, Rua das Estrelas, 4150-762 Porto, Portugal

19 Department of Physics and Astronomy, University of Missouri Kansas City, Kansas City, MO 64110, USA

20 Departamento de Astronomia do Instituto de Astronomia, Geofísica e Ciências Atmosféricas da USP, Rua do Matão 1226, Cidade Universitária, 05508-090 São Paulo, Brasil

21 Department of Physics and Astronomy, Macquarie University, NSW 2109, Australia

22 Max-Planck-Institut für Astronomie, Königstuhl 17, 69117 Heidelberg, Germany

23 CEI Campus Moncloa, UCM-UPM, Departamento de Astrofísica y CC. de la Atmósfera, Facultad de CC. Físicas, Universidad Complutense de Madrid, Avda. Complutense s/n, 28040 Madrid, Spain

24 Departamento de Investigación Básica, CIEMAT, Avda. Complutense 22, 28040 Madrid, Spain

25 Departamento de Física Teórica, Universidad Autónoma de Madrid, 28049 Madrid, Spain

26 Department of Physics, Royal Military College of Canada, PO Box 17000, Station Forces, Kingston, Ontario, K7K 7B4, Canada

27 European Southern Observatory, Alonso de Cordova 3107, Casilla 19001, Vitacura, Santiago, Chile 